\title{
Covid-19 fever symptom detection based on IoT cloud
}

\author{
Mustafa Wassef Hasan \\ Department of Electrical Engineering, University of Baghdad, Iraq
}

\section{Article Info \\ Article history: \\ Keywords: \\ Corona virus \\ Covid-19 \\ IoT \\ Node MCU \\ RFID \\ Temperature}

Received May 19, 2020

Revised Jul 22, 2020

Accepted Aug 10, 2020

\begin{abstract}
This paper presents a new method of detection Covid-19 fever symptoms depending on IoT cloud services to solve the higher time delay of checking the crowded clients that enter public or private agencies which can lead to a dangerous field to spread the disease. An automatically checking process is suggested using a practical experiment is developed using (ESP8266 node MCU, ultrasonic (SR-04), RFID (RC522), human body temperature (MAX30205) sensors, and ThingSpeak platform). Where node MCU is open-source hardware used to transmit the received data (human temperature sensor) from the (MAX30205) to the cloud platform (ThingSpeak) then alert the monitoring manager user when the collected data reached a critical value that specified previously and automatically take action to solve this situation. At the same time, the cloud platform will provide a graphical representation of the received data to display it using different monitoring devices such as (computers, mobiles, and others).
\end{abstract}

This is an open access article under the CC BY-SA license.

\section{Corresponding Author:}

Mustafa Wassef Hasan

Department of Electrical Engineering

University of Baghdad

Al-Jadriya, Baghdad, Iraq

Email: mustafawassef@yahoo.com

\section{INTRODUCTION}

In the recent years, the cloud platforms witnessed a significant development in terms of service provided and performance, which is used as one of the solutions to the problems experienced by the client in daily life for the business and public life. At the same time, many kinds of cloud service are introduced; some of them are paid. Others are for free, and many transmission models are used such as Bluetooth, Zigbee, Wifi...etc used for short-distance transmission and for long-distance communication such as Sigfox, LTE...etc these models transmit the data to the cloud that considered as a part of the internet of things (IoT). IoT defines relationships between human and devices, anything that related to technology so that allowing entirely or some of the devices to connect between each other and perform the needed job without requiring the human to interact.

Cloud services have been used as a monitoring platform that used alongside with external hardware like [1-3]. Cloud computing used with mobile devices [4, 5]. While other authors design a protocol based on key agreement protocols that help to secure data sharing and increase efficiency [6], a new study of cloud computing is studied in [7]. Some of the authors focused on virtualizing the smartboard (Arduino) in IoT cloud [8]. An IoT cloud used to monitor healthcare using Arduino microcontroller [9-11]. While on [12] used to control smart garden watering system.

This work-study the new pandemic coronavirus disease (Covid-19) fever detection method according to World Human Organization (WHO) one of the accompaniment symptoms is human body temperature increases above the normal $\left(37^{\circ} \mathrm{C}\right)$ [13-15]. Usually, a particular device used to measure the human temperature at airports, companies, hospitals, malls and many other locations. Still, sometimes it 
considers as a risky and tiring process to clients and monitoring manager users. The system model is a design based on esp8266 controller to receive and send data across other components and ThingSpeak cloud, such that the esp8266 controller connects with the cloud using Wi-Fi service. Ultrasonic (SR-04) is connected and works as motion detection to notify the system model whenever someone passes. RFID (RC522) used to identify the client information such as (name, time, date, and others). And finally, a temperature body sensor (MAX30205) used to measure the temperature of each client.

After that, an SMS message with a new notification sends to the monitoring manager used by the cloud when the system model detects a higher temperature. The remaining sections of this paper are formed as follows. Section 2 establishes the research method. In Section 3, result and discussion explained. Section 4 summarizes the conclusions and the key aspects of this research work.

\section{RESEARCH METHOD}

\subsection{ThingSpeak}

ThingSpeak launched by ioBridge in 2010 to support IoT applications, ThingSpeak is involved with MathWorks, which mean ThingSpeak supported from Matlab, and that leads to greatly help the users to analyze and visualize the data received by the sensors and recently used in many applications like [16-18]. Since 2010 ThingSpeak is growing due to flexibility in use and good performance. ThingSpeak is an opensource platform that related to internet of things (IoT) that used to store and recover the data by using several protocols. Such as hypertext transfer protocol which is known as (HTTP) that is responsible for data communication by another meaning user can access easily to other resources. The additional protocol is message queuing telemetry Transport which is known as (MQTT) that transports message between devices on remote areas. Figure 1 represents the connection of ThingSpeak with other things such as apps, web services, etc.

ThingSpeak connection

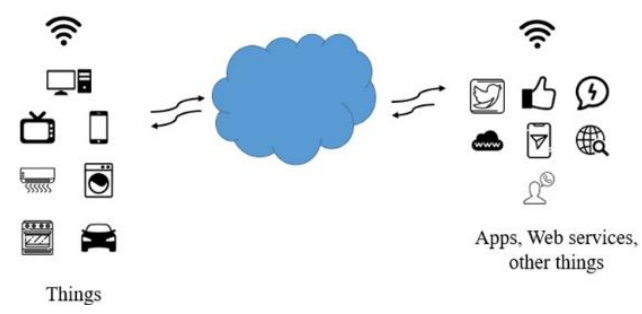

Figure 1. ThingSpeak connection

\subsection{Hardware}

\subsubsection{NodeMCU (ESP8266)}

NodeMCU is a low price sensor and an open-source (IoT) platform that used widely with (IoT) application such that used to control temperature and humidity environment like [19]. And with apps that used smart irrigation system [20]. NodeMCU processor can deal with (c/c++) like Arduino microcontroller. Which consist of (30) pins, and these pins can be assigned with different duties. Such it that include the (ADC channel) that are a (10-bit channel) or (PWM) or (UART interface) for serial code or (SPI, I2C and I2S interface) to connect the sensors and (I2S interface) for projects that add sound with (128 KB) memory. And storage of $(4 \mathrm{MBs})$ that used a (USB) to feed the board with power Figure 2. Show node-MCU controller.

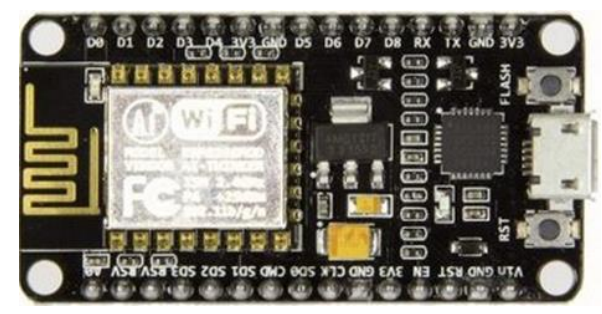

Figure 2. NodeMCU (ESP8266) controller 


\subsubsection{RFID (RC522)}

RFID (radio-frequency identification) typically consist of RFID reader and RFID tags; the primary mechanism of work depends on an electromagnetic field. Whenever the RFID tags nearby the RFID reader, the tags send digital data to read that can be used to identify clothes, goods, peoples, and many others. The tags identification range varies from a low range up to a long-range, RFID used in many applications such as [21-23]. RFID (RC522) module operate at 13.56 MHZ and support SPI, I2C, and UART and consist of (8) pins witch are (Vcc, RST, Ground, IRQ, MISO, MOSI, SCK, and SS) Figure 3 shows the RFID (RC522) and RFID tag sensors.

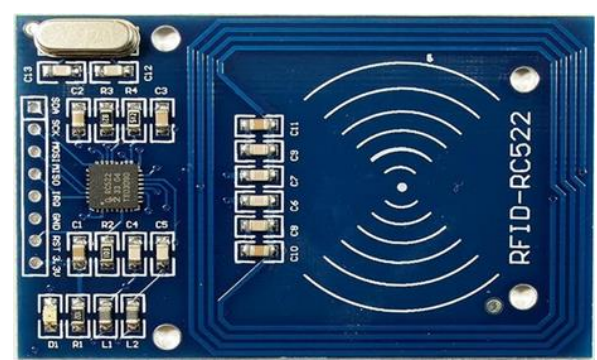

(a)

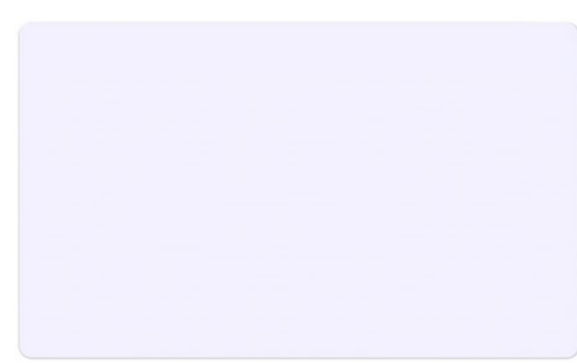

(b)

Figure 3. RFID sensor, (a) RFID (RC522), (b) RFID tag

\subsubsection{Ultrasonic (SR-04)}

Ultrasonic is a transceiver sensor that considers as distance sensor of an object that blocks the transmitted signal, and ultrasonic transceiver describe the sensor that sends an electrical signal and convert it into ultrasound energy and at the same time receive the ultrasound and turn it into an electrical signal. Ultrasonic (SR-04) range varies between $2 \mathrm{~cm}-400 \mathrm{~cm}$ and used widely in different application like [24-26]. At the same time, the (SR-04) consists of (4) pins which are (Vcc (power), Trig (send the signal), Echo (receive the signal), and GND (ground)) Figure 4 show ultrasonic (SR-04) sensor.

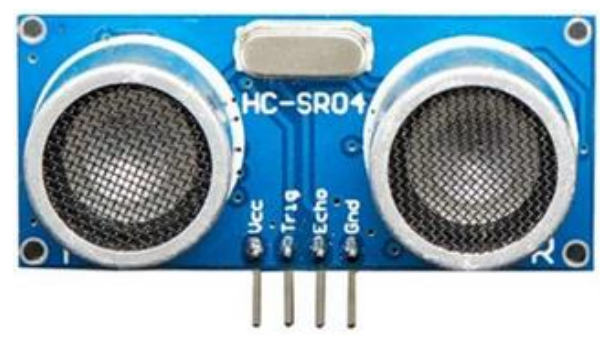

Figure 4. Ultrasonic (SR-04) scheme

\subsubsection{Human temperature sensor $\left(\mathrm{MAX30205}\left( \pm 0.1^{\circ} \mathrm{C}\right)\right)$}

Human body temperature (MAX30205) is a thermostat temperature that contributes a stable analytic body temperature of certainty of $0.1{ }^{\circ} \mathrm{C}\left(37{ }^{\circ} \mathrm{C}\right.$ to $\left.39{ }^{\circ} \mathrm{C}\right)$. With a resolution of 16 bits $\left(0.00390625^{\circ} \mathrm{C}\right)$, MAX3025 operates in $0^{\circ} \mathrm{C}$ to $+50^{\circ} \mathrm{C}$ temperature range where the sensor converts the measurements into digital readings using an analogue to digital converter (ADC), this sensor available as (8) pin package, Figure 5 shows typical application circuit. Table 1 shows the pin description of MAX30205.

Table 1. Pin description of MAX30205

\begin{tabular}{ccc}
\hline Pin & Name & Function \\
\hline 1 & VDD & 3.3V Supply Voltage Input. \\
2 & OS & Overtemperature Shutdown Output. \\
3 & SCL & Serial-Data Clock Input. \\
4 & SDA & Serial-Data Input/Output Line. \\
5 & GND & Ground \\
\hline
\end{tabular}




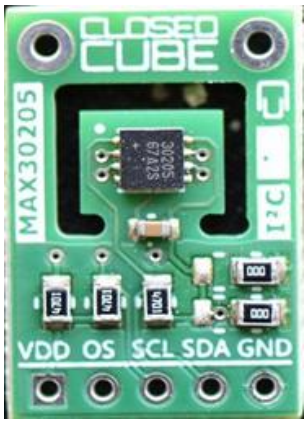

(a)

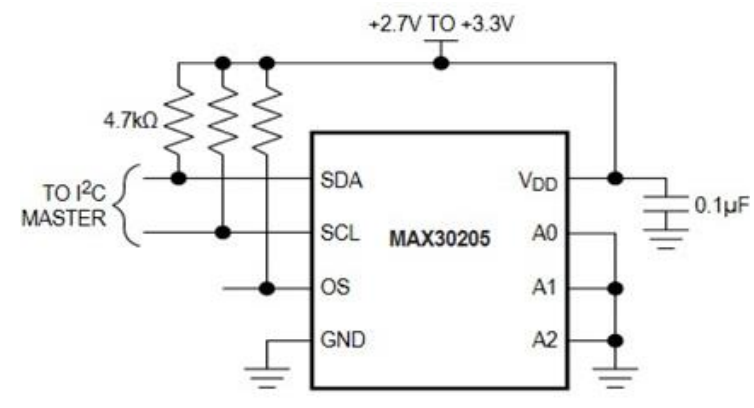

(b)

Figure 5. Typical application circuit, (a) general lock, (b) circuit connection

\subsection{System modelling}

The first procedure after assembling the hardware components mentioned above software is written to program system work, where ultrasonic (SR-04) is connected above an entrance door, such that when clients (users) try to enter the ultrasonic (SR-04) will sense this movement and send notification (flags) to (esp8266) to work. At the same time, the second procedure take action which related to the RFID (RC522) reader to sense the RFID (tags) that each client has, which contains the full information about the clients like (names, id number, address, etc.) that will be sent to (esp8266). The third procedure depends on temperature body sensor (MAX30205), where each client will put any finger of his hand on the (MAX30205) sensor. By then, the temperature of each entrance user is sent to the (esp8266) and check if it exceeds the allowed temperature $\left(37^{\circ} \mathrm{C}\right)$, an SMS message will be sent through the cloud (ThinSpeak) to the monitoring manager with all of the information of the user.

The fourth procedure is mainly responsible for modifying the (ThinSpeak) cloud to be suitable for executing the (SMS message) process, and this step performed using the (Apps) section in (ThingSpeak) which allow creating a (ThingHTTP and React) with external web service called (IFTTT). If This Then That known as (IFTTT) is a web service provide the required tools for the users to create (Applets) that can act in response to specific trigger action. Where in this case the (React app) works as a trigger of an HTTP request to the (IFTTT) applets to send the required message or notification automatically, many applications and researches have been used with (IFTTT) services like [27, 28]. Due to the touching of the (MAX30205) sensor by several clients, each client after entering the entrance door have to use antiseptic with a concentration of $75 \%$ alcohol to grantee no infection will transfer through the sensor. Figure 6 show the design scheme for the system model.

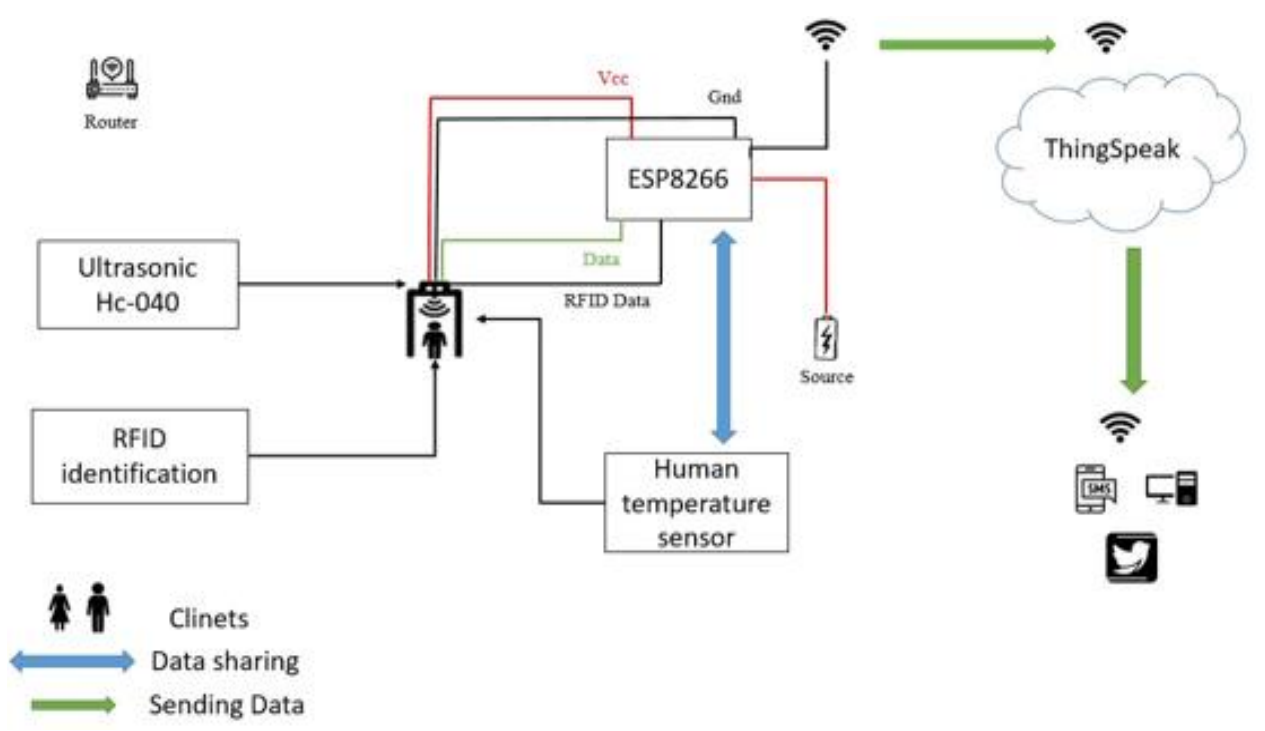

Figure 6. System model design 
Algorithm 1. Pseudocode of the system model

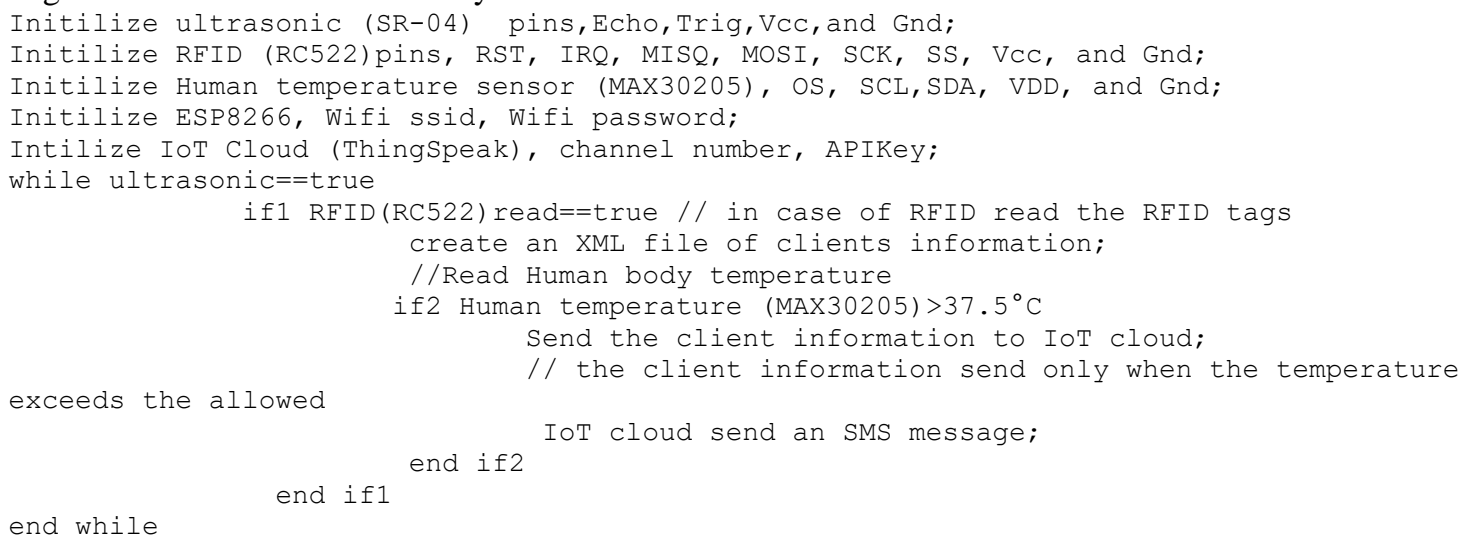

\section{RESULTS AND DISCUSSIONS}

After running the system model described in the previous section an Excel file created, that is connected to the (esp8266) to show the client attendance history which contains full information of the clients (date, time, name, and id number) with each one temperature measurement. The excel file is connected to the (esp8266) through (PLX DAQ Vxxx) program witch allow the excel sheet to communicate with any device supports the serial port protocol, originally developed to enable communication between excel sheet and Arduino. As shown in (8) RFID tags are used to experience the system model, where it refers to (8) clients, each one start to swipe his RFID tag and measure his temperature, the IoT cloud (ThingSpeak) start to receive each client data by then a database is created as shown in Figure 7.

Since there is no one from the clients exceeds $\left(38^{\circ} \mathrm{C}\right)$, it is assumed that the critical temperature allowed is to be $\left(37.5^{\circ} \mathrm{C}\right)$. As shown in Figure 7 , the client called (HUSSIEN.A) with id number (2453) reaches the critical temperature, such that immediately the cloud (ThingSpeak) will react through the (HTTP) and send a notification with an SMS message using (IFTTT) to the monitoring manager to alarm him with this situation. Figure 8 (a) shows a notification alarm reached the mobile device, while (b) shows the SMS message reached.

ThingSpeak is capable of representing the received data from the system model as graphical schemes to help the user for a better understanding Figure 9 show a continuous graphical representation for the (8) clients collected temperature. It clearly shows the critical temperature happens at (4:09) for (12) hour mode or (16:09) in (24) hour representation, while Figure 10 shows a discrete temperature representation.

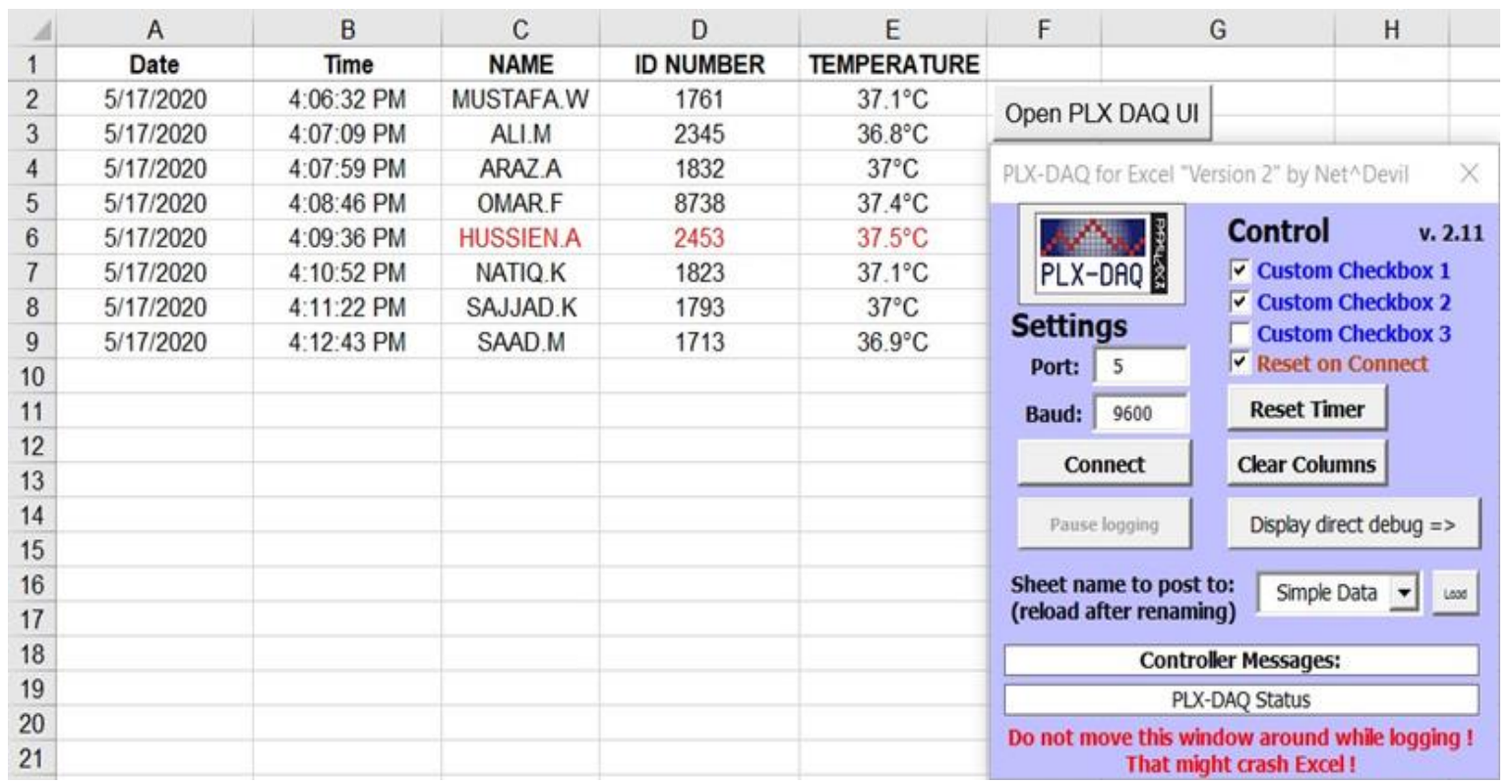

Figure 7. Clients database 
IFTTT 4:09 pm

IFTTT

"Higher-Temperature" detected equal to $37.5 \mathrm{c}$ of id number 2453 at May 17, 2020 at 4:09PM

(a)
Sunday, 17 May 2020

"Higher-Temperature" detected equal to $37.5 \mathrm{c}$ of id number 2453 at May 17, 2020 at 4:09PM

(b)

Figure 8. System monitoring instrument (a) notification received, (b) SMS message received

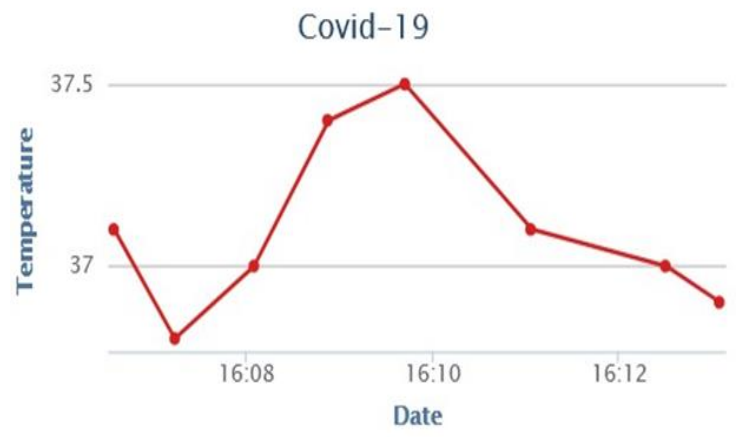

Figure 9. Continuous ThingSpeak temperature representation

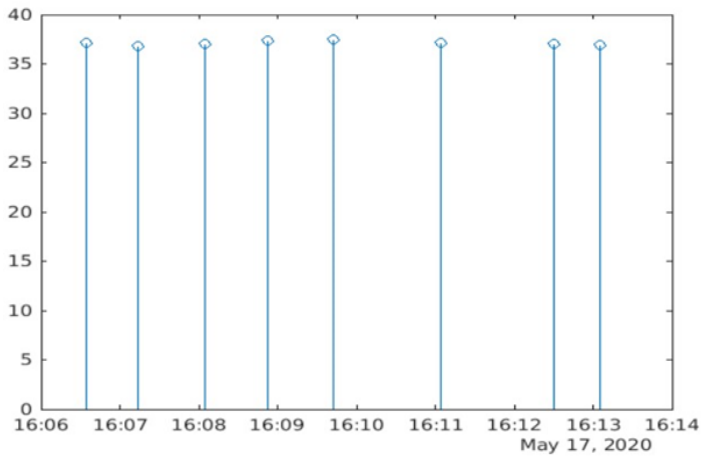

Figure 10. Discrete ThingSpeak temperature representation

\section{CONCLUSION}

A system model is a design to help to detect Covid-19 by identifying the fever symptoms of several clients enters private or public agencies. This model design consists of (esp8266 (Node-MCU), RFID (RC522), ultrasonic (SR-04), human temperature body (MAX30205)), ThingSpeak IoT cloud, user monitoring tools, IFTTT service. An SMS message with alarm notification sends to the monitoring manager with full data of client information when the system spot higher temperature that can be referred to a fever symptom of Covid-19. ThingSpeak cloud show a good and speed response to show the data received as a graphical representation. Future work is suggested to use another cloud platform that can be used to stream a live video of suspect cases with other social media applications.

\section{REFERENCES}

[1] D. Gupta, A. Parikh, and R. Swarnalatha, "Integrated healthcare monitoring device for obese adults using internet of things (IoT)," International Journal of Electrical and Computer Engineering (IJECE), vol. 10, no. 1, pp. 1239-1247, 2020.

[2] N. Kulkarni, S. V. N. L. Lalitha, and S. A. Deokar, "Real time control and monitoring of grid power systems using cloud computing," International Journal of Electrical and Computer Engineering (IJECE), vol. 9, no. 2, pp. 941-949, 2019.

[3] R. A. Rahman, U. R. Hashim, and S. Ahmad, "IoT based temperature and humidity monitoring framework," Bulletin of Electrical Engineering and Informatics (BEEI), vol. 9, no. 1, pp. 229-237, 2020.

[4] S. Abolfazli, Z. Sanaei, M. Alizadeh, A. Gani, and F. Xia, "An experimental analysis on cloud-based mobile augmentation in mobile cloud computing," IEEE Transactions on Consumer Electronics, vol. 60, no. 1, pp. 146-154, 2014.

[5] M. Shiraz, A. Gani, R. H. Khokhar, and R. Buyya, "A review on distributed application processing frameworks in smart mobile devices for mobile cloud computing," in IEEE Communications Surveys \& Tutorials, vol. 15, no. 3, pp. 1294-1313, 2013.

[6] J. Shen, T. Zhou, D. He, Y. Zhang, X. Sun, and Y. Xiang, "Block Design-Based Key Agreement for Group Data Sharing in Cloud Computing," in IEEE Transactions on Dependable and Secure Computing, vol. 16, no. 6, pp. 996-1010, 2019.

[7] S. T. Maguluri, R. Srikant, and L. Ying, "Heavy traffic optimal resource allocation algorithms for cloud computing clusters," Performance Evaluation, vol. 81, pp. 20-39, 2014.

[8] D. Bruneo, S. Distefano, F. Longo, G. Merlino, A. Puliafito, and A. Zaia, "Head in a Cloud: An approach for Arduino YUN virtualization," 2017 Global Internet of Things Summit (GIoTS), Geneva, 2017, pp. 1-6.

[9] S. Jayapradha and P. M. D. R. Vincent, "An IOT based human healthcare system using Arduino uno board," in 2017 International Conference on Intelligent Computing, Instrumentation and Control Technologies, Kannur, 2017, pp. 880-885.

[10] A. Kaur and A. Jasuja, "Cost Effective Remote Health Monitoring System Based on IoT Using Arduino UNO," Advances in Computer Science and Information Technology (ACSIT), vol. 4, no. 2, pp. 80-84, 2017. 
[11] P. Pavan and B. Balachander, "Emergency health care system using arduino uno," International Journal of Advanced Science and Technology, vol. 29, no. 3, pp. 5185-5188, 2020.

[12] A. Al-Omary, H. M. AlSabbagh, and H. Al-Rizzo, "Cloud based IoT for smart garden watering system using Arduino Uno," Smart Cities Symposium 2018, Bahrain, pp. 1-6, 2018.

[13] R. Madurai Elavarasan and R. Pugazhendhi, "Restructured society and environment: A review on potential technological strategies to control the COVID-19 pandemic," Science of The Total Environment, vol. 725, pp. 1-18, 2020.

[14] Z. Gao, et al., "A Systematic Review of Asymptomatic Infections with COVID-19," Journal of Microbiology, Immunology and Infection, 2020.

[15] M. Nicola, N. O'Neill, C. Sohrabi, M. Khan, M. Agha, and R. Agha, "Evidence based management guideline for the COVID-19 pandemic-Review article," International Journal of Surgery, vol. 77, pp. 206-216, 2020.

[16] A. H. Miry and G. A. Aramice, "Water monitoring and analytic based ThingSpeak," International Journal of Electrical and Computer Engineering (IJECE), vol. 10, no. 4, pp. 3588-3595, 2020.

[17] A. H. Ali, A. H. Duhis, N. A. Lafta Alzurfi, and M. J. Mnati, "Smart monitoring system for pressure regulator based on IOT," International Journal of Electrical and Computer Engineering (IJECE), vol. 9, no. 5, pp. 3450-3456, 2019.

[18] M. Swain, R. Singh, A. Gehlot, M. F. Hashmi, S. Kumar, and M. Parmar, "A reliable approach to customizing linux kernel using custom build tool-chain for ARM architecture and application to agriculture," International Journal of Electrical and Computer Engineering (IJECE), vol. 9, no. 6, pp. 4920-4928, 2019.

[19] D. A. Aziz, "Webserver Based Smart Monitoring System Using ESP8266 Node MCU Module," International Journal of Scientific and Engineering Research, vol. 9, no. 6, pp. 801-808, 2018.

[20] P. Srivastava, M. Bajaj, and A. S. Rana, "Overview of ESP8266 Wi-Fi module based smart irrigation system using IOT," 2018 Fourth International Conference on Advances in Electrical, Electronics, Information, Communication and Bio-Informatics (AEEICB), Chennai, 2018, pp. 1-5.

[21] A. Jamaluddin, D. Harjunowibowo, M. Akbar Rochim, F. Mahadmadi, H. Bulan Kakanita, and P. W. Laksono, "Implementation of RFID on Computer Based Test (RF-CBT) system," Proceedings of the Joint International Conference on Electric Vehicular Technology and Industrial, Mechanical, Electrical and Chemical Engineering (ICEVT \& IMECE), Surakarta, 2016, pp. 153-156.

[22] A. R. Laxmi and A. Mishra, "RFID based Logistic Management System using Internet of Things (IoT)," 2018 Second International Conference on Electronics, Communication and Aerospace Technology (ICECA), Coimbatore, 2018, pp. 556-559.

[23] P. Tan, H. Wu, P. Li, and H. Xu, "Teaching management system with applications of RFID and IoT technology," Education Sciences, vol. 8, no. 1, pp. 1-13, 2018.

[24] S. K. A. Nair, S. Joladarashi, and N. Ganesh, "Evaluation of ultrasonic sensor in robot mapping," $20193 \mathrm{rd}$ International Conference on Trends in Electronics and Informatics (ICOEI), Tirunelveli, India, 2019, pp. 638-641.

[25] A. Dimitrov and D. Minchev, "Ultrasonic sensor explorer," 2016 19th International Symposium on Electrical Apparatus and Technologies (SIELA), Bourgas, 2016, pp. 1-5.

[26] K. Manpreet and P. Jai, "Distance measurement of object by Ultrasonic Sensor HC-SR04," IJSRD-International Journal for Scientific Research \& Development, vol. 3, no. 5, pp. 503-505, 2015.

[27] R. K. Kodali and V. S. K. Gorantla, "RESTful Motion Detection and Notification using IoT," 2018 International Conference on Computer Communication and Informatics (ICCCI), Coimbatore, 2018, pp. 1-5.

[28] R. Xu, Q. Zeng, L. Zhu, H. Chi, X. Du, and M. Guizani, "Privacy Leakage in Smart Homes and Its Mitigation: IFTTT as a Case Study," IEEE Access, vol. 7, pp. 63457-63471, 2019. 